\title{
Populations and polypills: if yes, then how?
}

\author{
Richard S Cooper, ${ }^{1}$ Jennifer Layden ${ }^{1,2}$
}

For industrialised countries, control of cardiovascular disease (CVD) is the most important achievement of biomedicine in the last half-century-certainly in terms of decrease in disease burden. The orderly sequence of research, surveillance, trials and implementation organised by cardiovascular scientists has led to a decline of $80 \%$ for both coronary heart disease and stroke in the USA and other countries since the 1960s. ${ }^{12}$ One crucial reason has been the discovery of successful interventions across the entire sequence of events from the source of risk factors to the patient with disease (figure 1). The biggest impact, however, has come through prevention at the population level and-to a lesser extent-prevention among high-risk individuals; collectively, they account for about two-thirds of the decline. ${ }^{3}$ Based on analyses in the USA, it is sobering to recognise that aspirin alone contributed more to the fall in death rates than all surgical and catheter-based interventions combined. ${ }^{3}$ Prevention was achieved by a broad spectrum of methods, from change in food manufacture, improvements in individual eating patterns and antismoking campaigns to the use of drugs for persons with established risk factors. Nonetheless, millions of people continue to die from CVD and the challenge today is even more daunting, namely, how do we achieve the real goal, the elimination of CVD as a mass phenomenon? Reaching that goal, which for practical purposes we would define as less than $5 \%$ of deaths from CVD, will necessarily require more effective prevention.

Although primary prevention is still driving CVD rates down, the desired state of a population free of high blood pressure and elevated cholesterol is only a theoretical possibility. Pharmacological interventions will have to become more aggressive. In their well-written commentary, Holmes and Bhala provide a

${ }^{1}$ Department of Public Health Sciences, Loyola University Stritch School of Medicine, Maywood, Illinois, USA; ${ }^{2}$ Department of Medicine, Loyola University Stritch School of Medicine, Maywood, Illinois, USA

Correspondence to Dr Richard Cooper, Department of Public Health Sciences, Loyola Stritich School of Medicine, 2160 S. First Ave, Maywood, IL 60153, USA; rcooper@lumc.edu summary of the prospects and problems associated with the polypill. ${ }^{1}$ Mass treatment-or 'public health by pills'-is not entirely new; some campaigns at $\mathrm{TB}$ control involved mass treatment, and the topic was raised for discussion by one of us during the writing of the US Third Adult Treatment Panel guidelines for elevated cholesterol.

However, the polypill strategy is novel. Based on our schema in the figure, it falls somewhere between attempts to change 'adverse behavioural patterns' and treatment of 'persons with risk factors'. Locating it not only between sequential steps to disease but between the horizontal domains (ie, 'Pathway' and 'Target Population') was intentional; it does not fit naturally within any group of health providers or treatment modalities. In fact, we do not find Holmes and Bhala's principle analogy of the vaccine particularly apt. Vaccines provide individual protection but equally important is the reduction of aggregate burden of the infectious agent. The more typical off-hand metaphor is fortification of foods with micronutrients or putting chlorine in the water; and those comparisons are all made in jest.

The problem for the polypill is how to get it from the bottle in the store into the stomach of Everyman. ${ }^{4}$ Although they provide a useful discussion of the problem of adherence, we think the authors underplay the nature of that obstacle. We have extensive experience with non-prescription supplements and the vast majority are only taken for short intervals.
Consistent pill taking among persons with hypertension or hypercholesterolaemia, who make regular doctor visits, is somewhere between $15 \%$ and $50 \% .^{5}$ Electronic health records linking pharmacy dispensation to individual patients will improve adherence; however, it is not clear how that tool could be applied to the polypill. ${ }^{6}$ To be convincingly marketed, the polypill must be seen as a treatment to prevent heart disease. The polypill is not 'health promoting' in the sense of vitamins but disease preventing. Asymptomatic people, especially those who are young and men, are reluctant to start taking pills, since that act acknowledges a vulnerability that requires treatment. In fact, we sympathise with the urge to preserve a robust sense of physical well-being as long as possible. Maybe someday pills to prevent heart attacks will be as detached from the sickness role as vaccines, but that must a long way away.

The major problem with the polypill, therefore, is that the cultural framework within which its use would be implemented does not exist. It cannot be primarily doctor-driven, and it is unlikely to have more than a minimal impact if promoted through public health campaigns. Taking a medication is different from drinking skim milk or avoiding butter. We suspect many doctors will be unsupportive, if not downright hostile, especially when reports of side effects make the news. The polypill is unlikely to be very lucrative and will not flash on the TV screen during football games. We agree that we should move forward with this strategy but the nature of the obstacles will be new-if not unique-and current intervention methods may be sufficiently weak as to undermine the entire effort. On the other hand, in 1968 no one would have believed that we could cut deaths from CVD by $80 \%$. Prevention science has 
always been inventive; perhaps the perception of how to maintain good health will evolve quicker than we anticipate. Experience will soon answer that question. We wonder, however, whether the ultimate answer will still lie in moving further and further towards basic, old fashioned, healthy lifestyles. That, after all, has almost become the norm for the most educated and health conscious of our population. The polypill may well be a temporary crutch while we finish the job of prevention for all.

Contributors RSC conceived the article and JL assisted in writing.

Competing interests None.

Provenance and peer review Commissioned; internally peer reviewed.

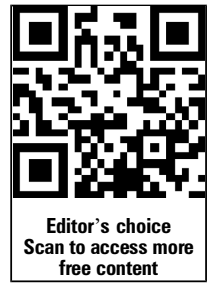

To cite Cooper RS, Layden J. J Epidemiol Community Health 2013;67:903-904.

Published Online First 6 July 2013

\section{(S) Linked}

http://dx.doi.org/10.1136/jech-2013-202690

J Epidemiol Community Health 2013;67:903-904. doi:10.1136/jech-2013-202949

\section{REFERENCES}

1 Holmes MV, Bahla. The physiological paradox: reframing the polypill as a vaccine for cardiovascular disease. JECH 2013.

2 Roger VL, Go AS, Lloyd-Jones DM, et al. Executive summary: heart disease and stroke statistics-2012 update: a report from the American heart association. Circulation 2012;125:188-97.

3 Ford ES, Ajani UA, Croft JB, et al. Explaining the decrease in U.S. deaths from coronary disease, 1980-2000. N Engl J Med 2007;356:2388-98.

4 Joyce J. Finnegans wake. Wordsworth Press, Reprinted 2012.

5 Wolf-Maier K, Cooper RS, Kramer K, et al. Hypertension treatment and control in five European countries, Canada and the U.S. Hypertension 2004:43:10-17.

6 Ram CV, Ramaswamy K, Qian C, et al. Blood pressure outcomes in patients receiving angiotensin II receptor blockers in primary care: a comparative effectiveness analysis from electronic medical record data. J Clin Hypertens 2011;11:801-12.

7 Cardiovascular Branch, Centers for Disease Control. A Public Health Plan to Prevent Heart Disease and Stroke. Atlanta, GA, 2003. 